\title{
Knockdown of IRX2 inhibits osteosarcoma cell proliferation and invasion by the AKT/MMP9 signaling pathway
}

\author{
TIELONG LIU ${ }^{1 *}$, WEIWEI ZHOU ${ }^{2 *}$, FEI ZHANG ${ }^{3 *}$, GUODONG SHI ${ }^{1}$, \\ HONGLIN TENG ${ }^{4}$, JIANRU XIAO ${ }^{1}$ and YAN WANG ${ }^{5}$
}

Departments of ${ }^{1}$ Orthopedics and ${ }^{2}$ Radiology, Changzheng Hospital, Second Military Medical University, Shanghai 200003;

${ }^{3}$ Department of Orthopedics, Ningbo Development Zone Center Hospital, Ningbo, Zhejiang 315800;

${ }^{4}$ Department of Orthopedics, First Affiliated Hospital, Wenzhou Medical University, Wenzhou,

Zhejiang 325000; ${ }^{5}$ Department of Orthopedics, Chinese People's Liberation Army

General Hospital, Beijing 100853, P.R. China

Received September 25, 2013; Accepted March 17, 2014

DOI: $10.3892 / \mathrm{mmr} .2014 .2215$

\begin{abstract}
Osteosarcoma is the most common type of human primary malignant bone tumor with a potential propensity for local invasion and distant metastasis. Thus, this study focused on the expression and roles of IRX2 in the development of osteosarcoma. The mRNA expression levels of IRX2 in tissue samples of 69 cases of human osteosarcoma were detected by a quantitative polymerase chain reaction assay. The associations between the expression levels of IRX2 and the pathological features of the tumor tissues were analyzed. Functional studies were performed by MTT and Matrigel invasion assays following IRX2 knockdown with a lentivirus vector. Western blotting was used to assay the protein expression levels of IRX2, p-AKT and matrix metalloproteinases (MMP). The results revealed that the expression levels of IRX2 were significantly increased in the primary human osteosarcoma tissues compared with those in the normal tissues, and the increase was significantly correlated with the tumor progression and prognosis of the patients. Furthermore, the proliferation and invasion of the cells were suppressed following IRX2 knockdown. Additionally, the mechanism by which IRX2 promoted cell proliferation and invasion by activating AKT and MMP9 was detected. In conclusion, these results indicated that IRX2
\end{abstract}

Correspondence to: Professor Jianru Xiao, Department of Orthopedics, Changzheng Hospital, Second Military Medical University, 415 Fengyang Road, Shanghai 200003, P.R. China

E-mail: jianru321xiao@163.com

Dr Yan Wang, Department of Orthopedics, Chinese People's Liberation Army General Hospital, 28 Fuxing Road, Beijing 100853, P.R. China

E-mail: yanwangarmy@163.com

*Contributed equally

Key words: osteosarcoma, IRX2, proliferation, invasion, prognosis promotes proliferation and invasion in osteosarcoma and implicated the potential of IRX2 in cancer therapy.

\section{Introduction}

Osteosarcoma is the most common type of highly malignant bone tumor in children and young adults, with a peak frequency during the second and third decades of life (1). Despite numerous therapeutic developments, including resection and effective multiagent chemotherapy, the five-year overall survival rate remained at $60 \%$ in $2011(2,3)$. However, $\sim 50 \%$ of patients face a poor outcome, particularly in those in which the osteosarcoma has metastasized to the lung (4-6). The precise molecular mechanisms that regulate the carcinogenesis, progression and metastasis of osteosarcoma remain poorly understood. Investigation of novel treatment strategies that effect tumor growth and metastasis is urgently required for treatment of osteosarcoma.

Osteosarcoma cells are characterized by unlimited cell growth and metastasis $(7,8)$. Thus far, increasing evidence has shown that the amplification of certain oncogenes is correlated with osteosarcoma tumor initiation and progression, including that of CD44, ERK5, ROCK1 and CCR5 (9-11). Identification of amplified oncogenes is becoming one of the methods with the most potential to reveal the molecular mechanisms contributing to osteosarcoma progression and metastasis. IRX2 is a member of the iroquois (Iro) and irx class of homeobox genes, which are important in the regionalization and patterning of tissues and organs during metazoan development. Studies have shown that IRX2 is amplified in numerous types of cancer, including acute lymphoblastic leukemia, breast cancer and soft tissue sarcomas (12-14). In addition to the identification of amplified IRX2 in numerous types of cancer, a study has demonstrated that the percentage of alterations in the DNA methylation of IRX2 in squamous cell carcinomas is as high as $85 \%$ (15). Little is known about the expression and potential functions of IRX2 in osteosarcoma pathophysiology and, in particular, in osteosarcoma metastasis.

The present study investigated the expression levels of IRX2 in osteosarcoma tissues compared with those in normal 
bone tissues, including in metastatic osteosarcoma tissues. Lentiviral-mediated IRX2 silencing was used to observe the effect of downregulated expression levels of IRX2 on cell proliferation, migration and invasion, in order to determine whether it is possible to target IRX 2 to offer a potential therapeutic application for osteosarcoma patients.

\section{Materials and methods}

Patients and osteosarcoma tissues. A total of 68 pairs of fresh and frozen osteosarcoma tumor tissues and the adjacent normal tissues were obtained from patients with histologically verified osteosarcoma who underwent surgical resection between 2004 and 2009 at Changzheng Hospital (Shanghai, China). All specimens were collected after obtaining written informed consent according to a protocol approved by the Ethics Committee of the Second Military Medical University (Shanghai, China). The study was performed according to the principles of the Declaration of Helsinki.

RNA extraction and quantitative polymerase chain reaction (qPCR) analysis. Total RNA was extracted from $25 \mathrm{mg}$ tissue or $5 \times 10^{5}$ cells using TRIzol reagent (Invitrogen Life Technologies, Carlsbad, CA, USA). A total of $500 \mathrm{ng}$ total RNA from each sample was reverse transcribed using PowerScript reverse transcriptase (Clontech Laboratories, Inc., Mountain View, CA, USA) according to the manufacturer's instructions. qPCR was performed using a FastStart SYBR Green Master kit (Roche Diagnostics GmbH, Mannheim, Germany) on an ABI Prism 7900HT Sequence Detection system (Applied Biosystems, Inc., Foster City, CA, USA). The following gene-specific primers were used for the qPCR: Forward, 5'-CGCCCTTCTACGGCAACTA-3' and reverse, 5'-CCCTCGCTGGCATCTTTCT-3' for IRX2; and forward, 5'-TTAGTTGCGTTACACCCTTTC-3' and reverse, 5'-GCTGTCACCTTCACCGTTC-3' for $\beta$-actin. The mRNA expression levels of IRX2 were analyzed using SDS software, version 2.3 (Applied Biosystems, Inc.) and normalized to those of $\beta$-actin mRNA, which was used as an internal control. The relative mRNA levels are presented as $\Delta \mathrm{Ct}$. Three independent experiments were completed and each reaction was performed in triplicate. All data are presented as the mean \pm standard deviation (SD).

Cell culture. The hFOB 1.19 human normal osteoblastic cell line (ATCC, Manassas, VA, USA) was maintained in Dulbecco's modified Eagle's medium/F-12 (1:1) with 10\% fetal bovine serum (FBS), $2.5 \mathrm{mM}$ L-glutamine (without phenol red) and $0.3 \mathrm{mg} / \mathrm{ml} \mathrm{G} 418$. The U2OS and SaOS2 human osteosarcoma cell lines were purchased from the American Type Culture Collection (Rockville, MD, USA) and were cultured in RPMI-1640 medium, which was supplemented with $10 \%$ FBS, $1 \%$ L-glutamine, $100 \mathrm{U} / \mathrm{ml}$ penicillin and $100 \mu \mathrm{g} / \mathrm{ml}$ streptomycin, in a humidified atmosphere containing $5 \% \mathrm{CO}_{2}$ at $37^{\circ} \mathrm{C}$. All cell lines were authenticated by Cancer Research UK (London, UK) in July 2010 using short tandem repeat profiling.

Plasmid construct and transduction. Specific IRX2 short hairpin RNA (shRNA) was designed to silence the expression of IRX2 (sense, 5'-CCGGCTACACAAACTACGGGAACTT CTCGAGAAGTTCCCGTAGTTTGTGTAGTTTTTG-3' and antisense, 5'-AATTCAAAAACTACACAAACTACGGGAA CTTCTC GAGAAGTTCCCGTAGTTTGTGTAG-3') and was cloned into the EcoRI and AgeI sites of the pLKO.1-TRC cloning vector plasmid (Addgene, Cambridge, MA, USA). The fireflyluciferasetargetsequenceCTTACGCTGAGTACTTCGA replaced the IRX2 target sequence as a control. The construction of the IRX2-shRNA was confirmed by DNA sequencing.

Lentiviral particles containing pLKO.1-anti-LUC shRNA (SCR) or pLKO.1-anti-IRX2 shRNA (shIRX2) were produced using FuGENE HD Transfection reagent (Roche Diagnostics $\mathrm{GmbH}$ ) according to the manufacturer's instructions. For infection, the U2OS and SaOS2 cells were grown in $25-\mathrm{cm}^{2}$ flasks and transduced at 40-50\% confluence with the lentiviral particles, in the presence of polybrene $(8 \mu \mathrm{g} / \mathrm{ml})$. After infection $(24 \mathrm{~h})$, the stable cells were selected in puromycin (Sigma-Aldrich, St. Louis, MO, USA) at a concentration of $5 \mu \mathrm{g} / \mathrm{ml}$ for $48 \mathrm{~h}$.

Cell proliferation and colony formation assays. Cells in the logarithmic growth phase $\left(3 \times 10^{3}\right.$ cells/well) were transferred to each well of a 96-well plate. The cell proliferation was evaluated by cell counting kit- 8 reagent (Dojindo, Kumamoto, Japan), at the time points of 0,3,6,9 and 12 days after seeding and the cells were incubated in the reagent at $37^{\circ} \mathrm{C}$ for $2 \mathrm{~h}$. The absorbance was read at $450 \mathrm{~nm}$ with a microplate reader (Bio-Rad Laboratories, Hercules, CA, USA). This assay was performed three times in triplicate.

A total of 500 cells from each group were suspended in $2 \mathrm{ml}$ culture medium and seeded in six-well plates for 14 days. The colonies were fixed and stained with $1 \%$ crystal violet for $20 \mathrm{~min}$ and then washed three times. The number of colonies with $>50$ cells in each well was counted as one colony. The experiment was repeated three times in triplicate.

Cell invasion assays. Transwell systems were coated with Matrigel (diluted 1:4) on the upper surface of the polycarbonic membrane (Costar; $8.0-\mu \mathrm{m}$ pore size; Corning Incorporated, Corning, NY, USA). Cells from each group were resuspended in serum-free RPMI-1640 media at a concentration of $3 \times 10^{5}$ cells $/ \mathrm{ml}$. An aliquot of $100 \mu \mathrm{l}$ cell suspension was added to the upper chamber and $600 \mu \mathrm{l}$ containing $10 \%$ FBS and RPMI-1640 was added to the lower chamber. Following incubation at $37^{\circ} \mathrm{C}$ in a $5 \% \mathrm{CO}_{2}$ incubator for $24 \mathrm{~h}$, the cells that had penetrated through the membrane were fixed with $4 \%$ paraformaldehyde and stained with $1 \%$ crystal violet. The invaded cells were washed and the upper surface of the membranes was wiped with a cotton-tipped applicator to remove the non-migratory cells. The invaded cells were counted in five non-overlapping fields and photographed, and the average numbers of cells of at least five fields from each well were assayed. Three independent assays were performed.

Western blot analysis. Cells from each group were lysed in radioimmunoprecipitation assay buffer (Cell Signaling Technology, Inc., Danvers, MA, USA) containing Complete Protease Inhibitor Cocktail tablets (one tablet=10 ml cocktail; Roche Diagnostics $\mathrm{GmbH}$ ). The protein expression levels of the cells were quantified using a bicinchoninic acid assay 
A

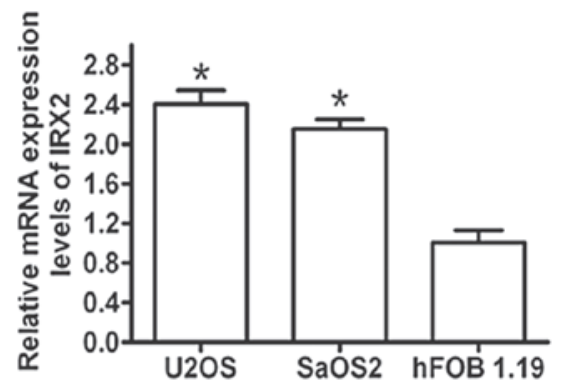

B

IRX2

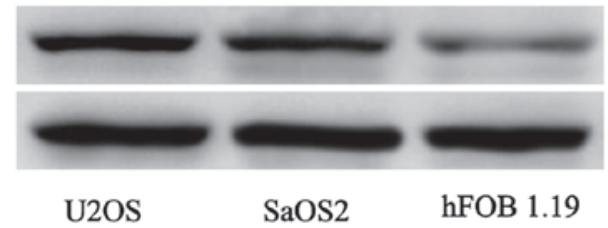

C

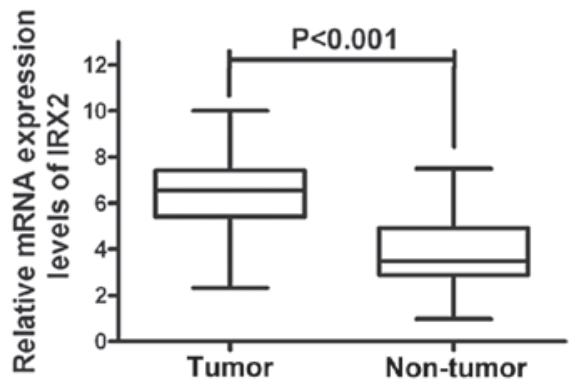

Figure 1. IRX2 expression levels were increased in the osteosarcoma cell lines and clinical human osteosarcoma tissues. (A) The IRX2 mRNA expression levels in two osteosarcoma cell lines (U2OS and SaOS2) and the hFOB 1.19 cell line. (B) The protein levels of IRX2 in the osteosarcoma cells and normal osteoblastic cells were determined by western-blotting, and $\beta$-actin was used as the internal loading control. (C) The $\Delta \mathrm{Ct}$ values of IRX2 in 68 paired clinical human osteosarcoma tissues and the adjacent normal tissues. ${ }^{*}<0.05$, compared with the relevant control.
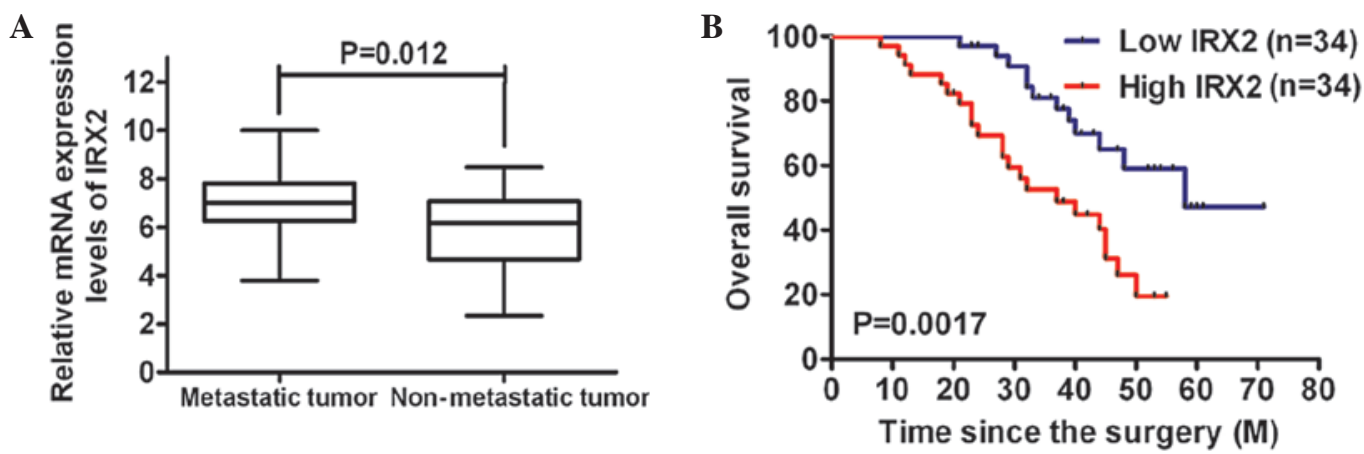

Figure 2. Association of IRX2 expression levels with metastasis and the overall survival rate. (A) Comparison of the mRNA expression levels of IRX2 between metastatic and non-metastatic osteosarcoma tissues. (B) Kaplan-Meier curves of the overall survival rate in the 68 osteosarcoma patients according to the IRX2 expression levels in the tumor tissues. The median value of IRX2 expression in these tumor samples was selected as the cut-off. The corresponding P-values analyzed by log-rank tests are indicated. Data were analyzed by SPSS software, version 15.0 with the P-value indicated.

(Sangon, Shanghai, China). Equal quantities of protein were separated on SDS-PAGE gels and transferred to a polyvinylidene difluoride membrane (Bio-Rad, Hercules, CA, USA), which was blocked using 6\% not-fat milk in Tris-buffered saline with $1 \%$ Tween-20. The membrane was incubated overnight at $4^{\circ} \mathrm{C}$ with primary antibodies (anti-IRX2 antibody, catalog code: ab72975; anti-MMP2 antibody, catalog code: 10373-2-AP; anti-AKT antibody, catalog code: \#9272; anti-p-AKT antibody, catalog code: \#9271; anti-MMP9 antibody, catalog code: \#3852; and anti-actin antibody, catalog code: CW0097). Following incubation, the membrane was washed and incubated for $1 \mathrm{~h}$ at room temperature with the appropriate horseradish peroxidase-conjugated secondary antibody (1:5,000; Kangcheng, Shanghai, China). The immunoblot images were all captured with an ImageQuant ${ }^{\mathrm{TM}}$ LAS-4000 (Fujifilm) and the chemiluminescence was directly analyzed and quantified with ImageQuant TL software (GE Healthcare, Little Chalfont, UK).

Statistical analysis. Data are presented as the mean \pm SD Statistical analyses were performed using two-tailed Student's t-test. Kaplan-Meier survival functions and log-rank test were used to assess the disease-free survival and overall survival times based on the mean expression levels of IRX2. All statistical analyses were two-sided and performed with SPSS software, version 15 (SPSS, Inc., Chicago, IL, USA). P<0.05 was considered to indicate a statistically significant difference.

\section{Results}

IRX2 expression levels are increased in osteosarcoma cells. In order to investigate the functions of IRX2 in human 
A

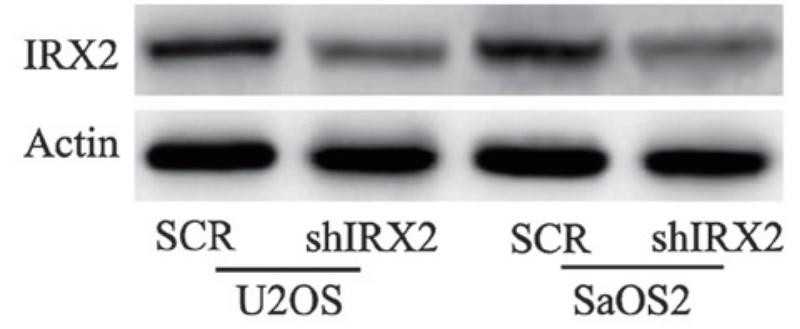

$\mathbf{C}$

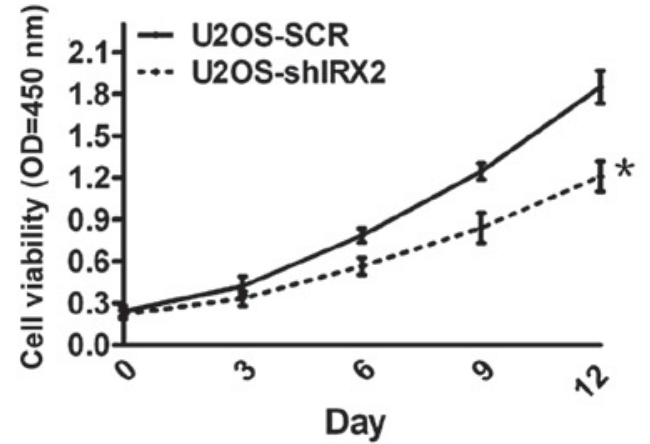

D

$\mathrm{U} 2 \mathrm{OS}$
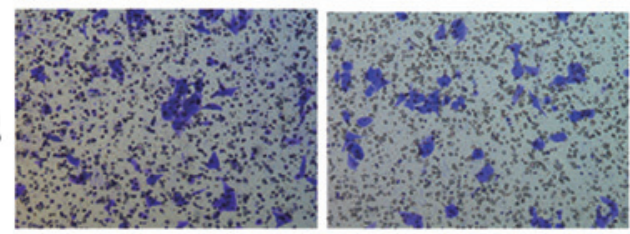

$\mathrm{SaOS} 2$

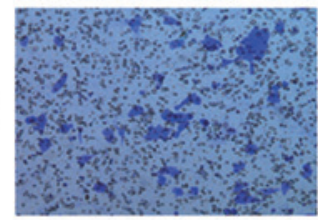

SCR

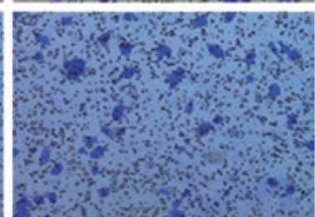

shIRX2
B
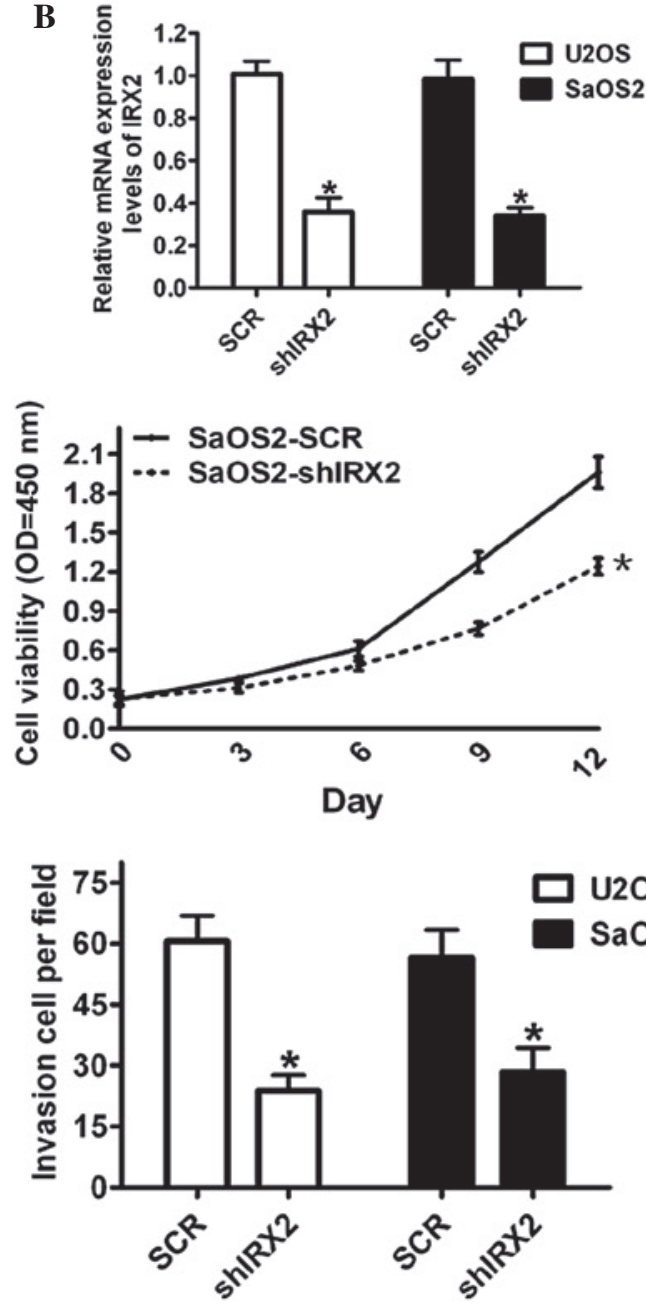

Figure 3. Effects of the knockdown of IRX2 expression levels on the growth and invasiveness of osteosarcoma cells. Downregulation of the IRX2 expression levels in U2OS and SaOS2 cells by transduction with shRNA-IRX2 was verified by (A) western blot analysis and (B) qPCR. (C) The proliferation of U2OS and $\mathrm{SaOS} 2$ cells. Significantly slower rates of proliferation were observed in the cells transduced with shIRX2 compared with those of the SCR-transduced tumor cells. (D) The migration and invasion analyses were visualized following staining with crystal violet and quantification of the number of invasive cells. Data are presented as the mean \pm standard deviation from three independent experiments. ${ }^{*} \mathrm{P}<0.05$, compared with the relevant control. SCR, pLKO.1-anti-LUC shRNA; shIRX2, pLKO.1-anti-IRX2 shRNA; OD, optical density; qPCR, quantitative polymerase chain reaction.

osteosarcoma development, the expression levels of IRX2 in the hFOB 1.19 human normal osteoblastic cell line were initially analyzed in comparison with those in the U2OS and $\mathrm{SaOS} 2$ human osteosarcoma cell lines by western blot analysis and qPCR. The protein and mRNA expression levels of IRX2 were significantly increased in the U2OS and SaOS2 osteosarcoma cells compared with those in the hFOB 1.19 normal osteoblastic cells (Fig. 1A and B).

Furthermore, the expression levels of IRX2 in the 68 pairs of human primary osteosarcoma tumor and adjacent normal tissue samples were assayed. As expected, the IRX2 expression levels were significantly increased in the tumor tissues compared with those in the paired adjacent non-cancerous tissues (Fig. 1C). These results indicated that the expression levels of IRX2 are increased in osteosarcoma cells and tissues, and IRX2 may be involved in human osteosarcoma development.

Upregulated IRX2 expression levels correlate with osteosarcoma metastasis and prognosis. Subsequently, it was determined whether the upregulation of IRX2 expression

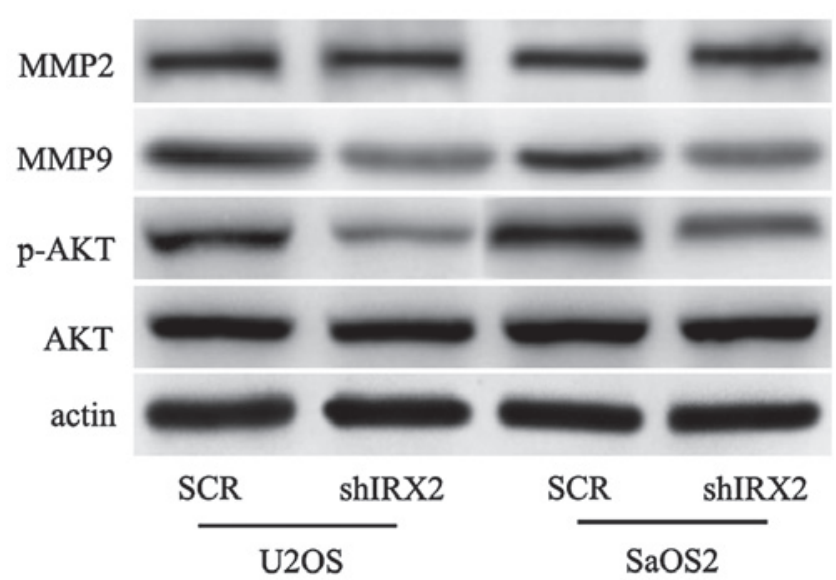

Figure 4. IRX2 regulated proliferation and migration of the cells through the AKT signaling pathway. The expression levels of MMP2, MMP9, p-AKT and AKT were detected by a western blotting assay using U2OS and SaOS2 cells after transduction with shIRX2 or SCR, and $\beta$-actin was used as the loading control. The image shown is representative of three independent experiments. MMP, matrix metalloproteinase; SCR, pLKO.1-anti-LUC shRNA; shIRX2, pLKO.1-anti-IRX2 shRNA. 
levels was correlated with the metastasis of osteosarcoma. The analysis of the mRNA expression levels of IRX2 identified that they were markedly increased in the metastatic tissues compared with those in the non-metastatic tissues (Fig. 2A). Furthermore, Kaplan-Meier analysis using the log-rank test was performed and the results revealed that the patients with low IRX2 expression levels in their osteosarcoma tumors had a longer median survival time of 58.0 months, compared with those with high IRX2 expression levels whose median survival time was 37.0 months (Fig. 2B). These findings suggested that the IRX2 expression levels increased with the aggressiveness of the tumors.

Silencing IRX2 reduces tumor cell growth and invasiveness in vitro. In order to study whether altered expression levels of IRX2 affect the functions of cells, a lentivirus-based RNAi delivery system with shRNAs against IRX2 was used in U2OS and $\mathrm{SaOS} 2$ cells (with high expression levels of endogenous IRX2) that are highly aggressive and exhibit a high metastatic potential. Efficient inhibition of IRX2 expression was confirmed by qPCR (Fig. 3A) and western blot analyses (Fig. 3B). In order to determine the effect of IRX2 on cell proliferation, an MTT assay was performed. The results showed that knockdown of the IRX2 expression levels had a significant inhibitory effect on the viability of the U2OS and SaOS2 cells compared with that of the control cells (Fig. 3C). Subsequently, the action of IRX2 on cell invasion was analyzed. The results demonstrated that IRX2 knockdown significantly reduced the invasiveness of the $\mathrm{U} 2 \mathrm{OS}$ and SaOS2 cells compared with that of the control group (Fig. 3D). These results showed that silencing IRX2 expression suppressed osteosarcoma cell proliferation, invasion and migration, suggesting that IRX2 is involved in bone tumor cell growth and invasiveness.

Involvement of $p$-AKT in IRX2-induced osteosarcoma cell proliferation and migration. Further experiments were conducted to reveal the underlying molecular mechanisms of the effect of IRX2 on osteosarcoma. The PI3K/Akt signaling pathway controls several cellular response functions, including proliferation, invasion, survival and metabolism. To explore whether PI3K/Akt signaling mediates IRX2-induced cell proliferation and invasion, this study investigated whether silencing IRX2 may reduce the expression levels of p-AKT. As shown in Fig. 4, the expression levels of p-AKT were markedly reduced following the knockdown of the expression levels of IRX2. Previous studies have shown that the upregulation of the expression levels of matrix metalloproteinases (MMPs) is a key step for promoting cell invasion (16). In the present study, the expression levels of MMP2 and MMP9 were determined by western blot analysis, and the results showed that the expression levels of MMP9 were markedly suppressed and that the expression levels of MMP2 were not clearly altered (Fig. 4). These data showed that the depletion of IRX2 in the U2OS and SaOS2 cell lines resulted in reduced levels of AKT activation and MMP9 protein.

\section{Discussion}

In the present study, the role of IRX2 in osteosarcoma cell growth and metastasis was investigated in vitro. The expression levels of IRX2 were determined in osteosarcoma and normal osteoblastic cells and the results indicated that IRX2 expression was elevated in the human osteosarcoma cells compared with the normal cells. To further confirm the elevated expression levels of IRX2 in osteosarcoma cells, the expression levels of IRX2 mRNA were compared between osteosarcoma tissue samples and the corresponding non-tumor tissue samples and the results demonstrated that the IRX2 mRNA expression levels were higher in the primary osteosarcoma tissue. Furthermore, the higher expression levels of IRX2 mRNA were significantly correlated with the clinical stage and status of the metastasis. The present findings that the expression levels of IRX2 are high in osteosarcoma tumors and correlate with the aggressiveness of osteosarcoma in humans supports targeting of IRX2 as an effective therapy in bone tumor development.

To investigate the functional role of IRX2 in osteosarcoma tumors, lentiviral-mediated IRX2 silencing was used in U2OS and SaOS2 cells that express high IRX2 levels in basal conditions. Specific knockdown of the IRX2 expression levels markedly suppressed cell growth, which suggested that IRX2 is involved in cell proliferation in osteosarcoma cells. Metastasis is the major cause of human cancer-related mortalities and controlling metastasis is a key element that is possible to exploit therapeutically. The present study showed that knockdown of the IRX2 expression levels inhibited osteosarcoma cell invasion and migration, which are hallmarks of tumorigenesis. These data suggested that IRX2 is important in the cell growth and invasion of human osteosarcoma cells.

With regards to the mechanisms involved in cell growth and invasion, the expression levels of p-AKT were detected as the activation of $\mathrm{p}-\mathrm{AKT}$ promotes cell motility and invasion in numerous types of cancer cell (17-19). As expected, the levels of p-AKT were downregulated following inhibition of IRX2 using the lentivirus vector. Furthermore, IRX2 was detected as a key molecule in the regulation of the expression of MMP9, but not in that of MMP2. MMP9 is considered to be a marker of highly metastasizing cancers and the expression levels of MMP9 are involved in an aggressive, advanced and invasive or metastatic tumor phenotype $(20,21)$.

On the basis of these findings, the present study demonstrated high expression levels of IRX2 in osteosarcoma cells and tumor tissues, and, to the best of our knowledge, provided the first evidence that IRX2 depletion inhibits osteosarcoma cell growth and invasion in vitro. These findings suggested that IRX2 may be a potential therapeutic target for osteosarcoma treatment and additional mechanisms of IRX 2 remain to be elucidated in future studies.

\section{References}

1. Kansara M and Thomas DM: Molecular pathogenesis of osteosarcoma. DNA Cell Biol 26: 1-18, 2007.

2. Broadhead ML, Clark JC, Myers DE, Dass CR and Choong PF: The molecular pathogenesis of osteosarcoma: a review. Sarcoma 2011: 959248, 2011.

3. Khanna C, Wan X, Bose S, et al: The membrane-cytoskeleton linker ezrin is necessary for osteosarcoma metastasis. Nat Med 10: 182-186, 2004

4. Zhang P, Yang Y, Zweidler-McKay PA and Hughes DP: Critical role of notch signaling in osteosarcoma invasion and metastasis. Clin Cancer Res 14: 2962-2969, 2008. 
5. Su Y, Wagner ER, Luo Q, et al: Insulin-like growth factor binding protein 5 suppresses tumor growth and metastasis of human osteosarcoma. Oncogene 30: 3907-3917, 2011.

6. Zhang P, Yang Y, Zweidler-McKay PA and Hughes DP: Critical role of notch signaling in osteosarcoma invasion and metastasis. Clin Cancer Res 14: 2962-2969, 2008.

7. Ando T, Ichikawa J, Okamoto A, Tasaka K, Nakao A and Hamada Y: Gemcitabine inhibits viability, growth, and metastasis of osteosarcoma cell lines. J Orthop Res 23: 964-969, 2005.

8. Kim SM, Lee H, Park YS, Lee Y and Seo SW: ERK5 regulates invasiveness of osteosarcoma by inducing MMP-9. J Orthop Res 30: 1040-1044, 2012.

9. Gvozdenovic A, Arlt MJ, Campanile C, et al: CD44 enhances tumor formation and lung metastasis in experimental osteosarcoma and is an additional predictor for poor patient outcome. J Bone Miner Res 28: 838-847, 2013.

10. Liu X, Choy E, Hornicek FJ, et al: ROCK1 as a potential therapeutic target in osteosarcoma. J Orthop Res 29: 1259-1266, 2011

11. Wang SW, Wu HH, Liu SC, et al: CCL5 and CCR5 interaction promotes cell motility in human osteosarcoma. PLoS One 7: e35101, 2012.

12. Kang H, Wilson CS, Harvey RC, et al: Gene expression profiles predictive of outcome and age in infant acute lymphoblastic leukemia: a Children's Oncology Group study. Blood 119: 1872-1881, 2012.

13. Kadota M, Sato M, Duncan B, et al: Identification of novel gene amplifications in breast cancer and coexistence of gene amplification with an activating mutation of PIK3CA. Cancer Res 69 7357-7365, 2009
14. Adamowicz M, Radlwimmer B, Rieker RJ, et al: Frequent amplifications and abundant expression of TRIO, NKD2, and IRX2 in soft tissue sarcomas. Genes Chromosomes Cancer 45: 829-838, 2006.

15. Rauch TA, Wang Z, Wu X, Kernstine KH, Riggs AD and Pfeifer GP: DNA methylation biomarkers for lung cancer. Tumour Biol 33: 287-296, 2012.

16. Stamenkovic I: Matrix metalloproteinases in tumor invasion and metastasis. Semin Cancer Biol 10: 415-433, 2000.

17. Xue G, Restuccia DF, Lan Q, et al: Akt/PKB-mediated phosphorylation of Twist1 promotes tumor metastasis via mediating cross-talk between PI3K/Akt and TGF- $\beta$ signaling axes. Cancer Discov 2: 248-259, 2012.

18. Teranishi F, Takahashi N, Gao N, et al: Phosphoinositide 3-kinase inhibitor (wortmannin) inhibits pancreatic cancer cell motility and migration induced by hyaluronan in vitro and peritoneal metastasis in vivo. Cancer Sci 100: 770-777, 2009.

19. Fang Y, Xue JL, Shen Q, Chen J and Tian L: MicroRNA-7 inhibits tumor growth and metastasis by targeting the phosphoinositide 3-kinase/Akt pathway in hepatocellular carcinoma. Hepatology 55: 1852-1862, 2012.

20. Lu Y and Wahl LM: Production of matrix metalloproteinase-9 by activated human monocytes involves a phosphatidylinositol-3 kinase/Akt/IKKalpha/NF-kappaB pathway. J Leukoc Biol 78: 259-265, 2005

21. Gustin JA, Ozes ON, Akca H, et al: Cell type-specific expression of the IkappaB kinases determines the significance of phosphatidylinositol 3-kinase/Akt signaling to NF-kappa B activation. J Biol Chem 279: 1615-1620, 2004 